Table 1

Mean Responses Per Interval and Successive Quarter of Interval Subsequent to Rewarded and Nonrewarded Trials for All Three Ss Based on Five Experimental Sessions

\begin{tabular}{|c|c|c|c|c|c|c|}
\hline & & \multicolumn{4}{|c|}{ Interval Segment } & \multirow{2}{*}{$\begin{array}{c}\text { Interval } \\
\text { Total }\end{array}$} \\
\hline & & 1 & 2 & 3 & 4 & \\
\hline$S-M$ & $\begin{array}{l}\text { Reward } \\
\text { Nonreward }\end{array}$ & $\begin{array}{r}.75 \\
2.92\end{array}$ & $\begin{array}{l}1.53 \\
3.24\end{array}$ & $\begin{array}{l}2.48 \\
3.40\end{array}$ & $\begin{array}{l}4.25 \\
4.20\end{array}$ & $\begin{array}{r}9.01 \\
13.76\end{array}$ \\
\hline $\mathbf{S}-\mathbf{H}$ & $\begin{array}{l}\text { Reward } \\
\text { Nonreward }\end{array}$ & $\begin{array}{r}.08 \\
2.36\end{array}$ & $\begin{array}{r}.16 \\
2.64\end{array}$ & $\begin{array}{r}.50 \\
1.76\end{array}$ & $\begin{array}{l}1.95 \\
3.56\end{array}$ & $\begin{array}{r}2.69 \\
10.32\end{array}$ \\
\hline$S-G$ & $\begin{array}{l}\text { Reward } \\
\text { Nonreward }\end{array}$ & $\begin{array}{r}.92 \\
1.30\end{array}$ & $\begin{array}{l}1.68 \\
2.00 \\
\end{array}$ & $\begin{array}{l}2.10 \\
1.56 \\
\end{array}$ & $\begin{array}{l}3.17 \\
2.50\end{array}$ & $\begin{array}{l}7.87 \\
7.36 \\
\end{array}$ \\
\hline
\end{tabular}

following rewarded and nonrewarded trials for S-M, while for $S-G$ the responses following reward actually exceeded responses following nonreward. In terms of total number of responses across the 1 -min interval, $\mathrm{S}-\mathrm{H}$ and $\mathrm{S}-\mathrm{M}$ showed a general increment in responding ( $283 \%$ and $53 \%$, respectively), while S-G showed no overall effect, the increase early in the interval being balanced by a subsequent decrement in the later half of the interval for this $S$. Combining the percentage increases for all three Ss, an overall increase of $110 \%$ is seen following nonrewarded trials.

The behavior of all three Ss in the first $30 \mathrm{sec}$ after nonreward is consistent with frustration theory predicting response enhancement following nonreward. These findings are consistent with similar FI operant studies with animals (Staddon \& Innis, 1966). Davenport \& Thompson (1965) also found similar response enhancement following nonreward in a free-operant FR procedure. As in previous studies with children (Ryan \& Watson, 1968), the immediate effect is of greatest magnitude. Interestingly, the effect continued for a greater length of time than the usual 10-sec period demonstrated in other work. Such discrepancies are likely a function of the schedule and are consistent with the prediction that, where uncontaminated with response ceilings, an energizing effect of greater than $10-\mathrm{sec}$ duration might be evidenced. These findings extend frustration findings to a profoundly retarded population.

It may be argued that the effects are explicable purely on the grounds that following rewarded trials $S$ had an M\&M to manipulate, while on frustrated trials he did not, hence he manipulated the plunger. Observational data indicated that all Ss immediately ate the $M \& M$, having it in mouth before the end of the 5 -sec reinforcement event. The suppression effect of rewarded trials has been discussed by Wagner (1959) and FE demonstrated with such effects well-controlled.

That the FE was evident in the later quarter of the interval for only one of three Ss may suggest its dissipation. Since responding in the FI is also an increasing change at reinforcement was dramatic and significantly defined the reinforcement event; however, this analysis remains relevant.

While the FI offers interesting possibilities for frustration research, many questions remain unanswered. Studies in process by the author relate to the issues as defined above.

\section{REFERENCES}

AMSEL, A. The role of frustrative non-reward in noncontinuous reward situations. Psychological Bulletin, 1958, 55, 102-119.

function of time since rcinforcement and responding following rewarded trials was relatively high at this point, the final quarter may suffer from the response ceiling problem.

Another question arising from this investigation involves the similarity of nonreward conditions as defined in this experiment and the typical secondary reinforcement situation. Stimuli occurring on nonrewarded trials were the same as those paired with reinforcement on rewarded trials, hence nonreward consisted of secondary reinforcement. That most of the frustration literature involves this same similarity has been noted by Longstreth (1966). With such considerations in mind, an incentive motivation interpretation is also relevant to the data.

A final problem involves the special characteristics of the interval schedule. Time since reinforcement is a significant variable related to response probability in the interval schedule, hence increased rate of responding following nonreward may in fact be a function of time since reward. Staddon (1967) suggests a similar argument for the animal FI data. The stimulus

A ENPORT, J. W., \& THOMPSON, C. I. The Amsel frustration effect in monkeys. Psychonomic Science, 1965, 3, 481-482.

LONGSTRETH, L. E. Frustration and secondary reinforcement concepts as applied to human instrumental conditioning and extinction. Psychological Monographs: General \& Applied, $1966,80,29$.

RYAN, T. J., \& WATSON, P. Frustrative non-reward theory applied to children's behavior. Psychological Bulletin, 1968, 69, 111-125.

SCHOENFELD, W. N., CUMMING, W. W., \& HEARST, E. On the classification of reinforcement schedules. Proceedings of the National Academy of Sciences, 1956, 42, 563-570. SPRADLIN, J. E., GIRARDEAU, F. L., \& CORTE, E. Fixed ratio and fixed interval behavior of severely and profoundly retarded subjects. Journal of Experimental Child Psychology, 1965, 2, 340-353.

STADDON, J. E. R. Attention and temporal discrimination: Factors controlling responding under a cyclic-interval schedule. Journal of the Experimental Analysis of Behavior, 1967, 10, 349-359.

STADDON, J. E. R., \& INNIS, N. K. An effect analogous to "frustration" on interval reinforcement schedules. Psychonomic Science, 1966, 4, 287-288.

WAGNER, A. $R$. The role of reinforcement and non-reinforcement in an apparent frustration effect. Journal of Experimental Psychology, $1959,57,130-136$

\title{
Instructions to ignore a stimulus and the GSR
}

\section{ROBERT J. KOHLENBERG, University of notion that effects due to signal value are Washington, Seattle, Wash. 98105 \\ larger than effects due to demand characteristics.}

According to research on the orienting reflex, the instructions to ignore a stimulus would increase the signal value and result in an increased GSR elicited by that stimulus. A decreased GSR, however, would be expected due to the effect of demand characteristics of the experiment. As a test of these opposing effects, Ss were presented three different tones and instructed to ignore one of them. The results indicate that the GSR is enhanced for the ignored stimulus and support the
This study is concerned with the instruction to "ignore" a stimulus on the elicited GSR. Such effects would be important in the interpretation of studies in which the experimental effect is measured by reduced GSRs if a $S$ could produce a reduced GSR voluntarily as a result of $E$ hypothesis (Rosenthal, 1963) or demand characteristics (Orne, 1962).

The GSR is a frequently employed dependent variable in a wide range of 
psychological research. Some of the attraction to the use of GSR is a result of its autonomic involuntary properties. That is, if the response is not under voluntary control, the GSR would be less susceptible to a S's inclination to "psyche" out the experiment or conform to the E's expectancy (Orne, 1962; Rosenthal, 1963).

Research involving the GSR as a measure of the orienting reflex (Sokolov, 1963) does indicate, however, that the magnitude of the GSR can be increased through voluntary behavior. Any operation that results in an increase in the signal value of the stimulus being presented results in an increased magnitude of the GSR. According to Sokolov, the signal value of a stimulus can be enhanced through temporary connections via the second signal system. An example of enhancement of GSR via temporary connections has been reported by Maltzman \& Raskin (1965). These investigators instructed different experimental groups to press a foot pedal, to count, or to free associate implicitly whenever they heard the word "light." The control group was instructed to sit quietly and listen. The word "light" was imbedded in a sequentially presented list of stimulus words. The magnitude of GSR and resistance to extinction with repeated presentations (habituation) was greater when the word "light" was presented than for other stimulus words in the experimental groups. These results would suggest that experimental procedures that differentiate one stimulus from others could result in an increased signal value and corresponding increased magnitude ' of GSR elicited by that stimulus.

The concern of this current study is not the voluntary enhancement of GSR as Maltzman and Raskin obtained but, instead, the voluntary attenuation of GSRs. Research currently being conducted in this laboratory employs an experimental treatment in which one stimulus is differentiated from all other stimuli, but whose effectiveness is measured by a reduction in the magnitude of the GSR to the experimental stimulus. A similar experimental effect has been reported by Dean, Randall, \& Streiner (1968), who paired overtly or implicitly verbalized stimuli with the cessation of shock and subsequently tested for inhibiting effects on the GSR. Habituation, a basic finding in research involving the GSR, is also a result measured by reduced GSRs. The demand characteristics in such experiments required reduction in GSR. It can thus be argued that the reduced GSR is a result of the demand characteristics and not the specific experimental treatments that would result in GSR reduction. In the present study, the instruction administered was to ignore one stimulus of a series of stimuli that were presented to the $S$. The effects of the demand characteristics on the GSR elicited by the ignored stimulus would be an inhibition of the GSR, whereas the added signal value of the stimulus would result in enhancement.

\section{METHOD}

Twelve students from introductory psychology classes (six men and six women) served as Ss. Three stimuli, a low-pitch 500-cycle, a medium-pitch 600-cycle, and a high-pitch 700-cycle tone at $75 \mathrm{~dB}$ were presented in the sequence of low, medium, high, low, medium, high, etc., for a total of 18 presentations. The stimuli were presented at an interstimulus interval of $20 \mathrm{sec}$ on a tape recording and delivered through earphones to the S. GSR was recorded through finger electrodes on a Physiograph DMP-4A. The Ss were informed that three stimuli of low, medium, and high pitch would be repeatedly presented to them. They were then instructed to ignore the low-pitch tone (four Ss), the medium-pitch tone (four Ss), or the high-pitch tone (four Ss). The instructions also stated that the electrodes were used to measure reactions to the stimuli and that ignoring the stimulus would reduce the reaction.

\section{RESULTS}

Since novelty, a condition that elicits an orienting reflex, was maximal on the first presentation of each tone, analyses were conducted on the responses elicited by the second and following presentations of the ignored and other two stimuli. The GSRs elicited by the ignored stimulus, the

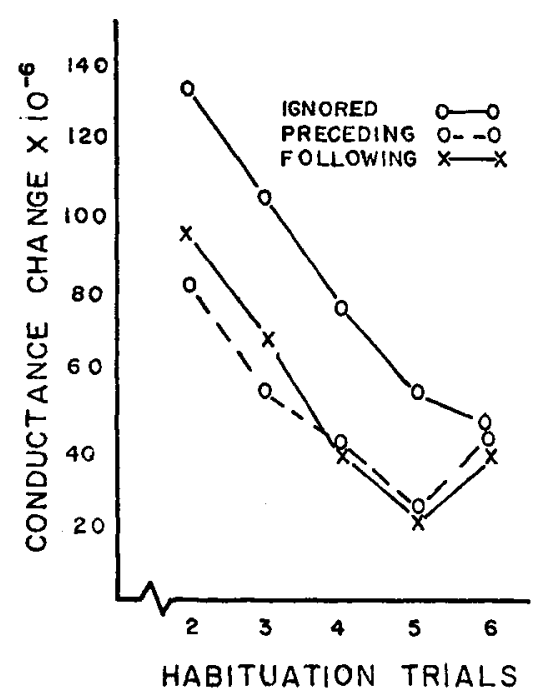

Fig. 1. GSR in conductance change units for the second through sixth presentation of the experimental stimuli. immediately preceding, and the immediately following stimulus are given in Fig. 1. An analysis of variance indicated that the GSR elicited by the ignored (critical) stimulus was reliably greater than the GSR elicited by the preceding and the following stimulus $(F=6.08, \mathrm{df}=2 / 154$, $\mathrm{p}<.05)$. There was no reliable interaction between the stimuli and trials.

\section{DISCUSSION}

The results indicate that the instruction to ignore a stimulus resulted in an enhanced value of the GSR elicited by that stimulus. The instructions in this experiment could have affected the GSR elicited by the critical stimulus in two ways: (1) increased magnitude due to signal value, and (2) decreased magnitude due to demand characteristics. The results suggest that the effects of the former are greater than those of the latter. The results also support the notion that in experiments in which one stimulus is differentiated from other stimuli and that stimulus results in reduced magnitudes of GSR, treatment effects other than demand characteristics are responsible for the reduced GSR. It appears that demands for inhibition of the GSR have the opposite effect-enhancement of GSR magnitude.

It could be argued that the $S s$ in this study hypothesized that the effects desired by the $E$ were the opposite of those stated in the instructions and conformed to this hypothesis by producing larger GSRs to the ignored stimulus. Such an interpretation is always possible when one attempts to stay ahead of the $S$ in terms of hypothesis making. It would appear, however, that evaluating the effect of directly stated demands on voluntary inhibition of the GSR is a first step in answering the general question posed in this paper.

\section{REFERENCES}

DEAN, S. J., RANDALL, B., \& STREINER, D. Mediational control of the GSR. Journal of Experimental Research in Personality, 1968, 3 , 71-76.

MALTZMAN, I., \& RASKIN, D. Effects of individual differences on the orienting reflex. Journal of Experimental Research in Personality, 1965, 1, 1-16.

ORNE, M. T. On the social psychology of the psychological experiment: With particular reference to demand characteristics and their implications. American Psychologist, 1962, 17, 714-783.

ROSENTHAL, R. On the social psychology of the psychological experiment: The experimenter's hypothesis as unintended determinant of experimental results. American Scientist, 1963, 51, 268-283.

soKOLOV, E. N. Perception and the conditioned reflex. New York: Macmillan, 1963.

\section{NOTE}

1. This research was supported in part by the University of Washington Graduate School Research Fund, Grant 11-7105. 